\title{
PALMITO DE PEJIBAYE (Bactris gasipaes K.): DISTANCIAS DE SIEMBRA Y MANEJO DE MALEZAS 1
}

\author{
Antonio Bogantes ${ }^{2}$, Renán Agüero ${ }^{3}$, Jorge Mora ${ }^{4}$
}

\begin{abstract}
RESUMEN
Palmito de pejibaye (Bactris gasipaes $\mathrm{K}$.): distancias de siembra y manejo de malezas. El trabajo se realizó en la Estación Experimental Los Diamantes, ubicada en Guápiles, Costa Rica. Se evaluó el efecto de cuatro densidades de siembra $(20.000,10.000,6.666$ y 5.000 plantas/ha) y control de malezas, física y química, sobre el crecimiento y el rendimiento inicial de una variedad de pejibaye sin espinas (Diamantes 10). Las variables evaluadas fueron: altura de la planta, grosor del tallo, número de brotes por planta; producción de palmitos, rendimiento industrial y económico. Además, se midió el efecto de la libre competencia del pejibaye con las malezas sobre el crecimiento de las plantas durante los primeros meses del cultivo. Se observaron diferencias entre distancias en la altura de la planta y en el número de brotes por cepa nueve meses después de la siembra. Ambas variables alcanzaron su mayor valor cuando la densidad fue de 6.666 plantas/ha $(0,75 \mathrm{~m}$ x 2 $\mathrm{m})$. Las mayores densidades de siembra produjeron los mejores rendimientos, en número de palmitos cosechados y número de cajas de exportación, durante los primeros cuatro meses de producción.
\end{abstract}

Palabras claves: pejibaye, Bactris gasipaes, palmito, cultivo, densidades.

\begin{abstract}
Growth and initial yield under four planting densities and two weed managing strategies of peach palm (Bactris gasipaes K.) for palm hearts. The Study was conducted at Los Diamantes Experiment Station, located in Guápiles, Costa Rica. Four planting densities of the spineless variety "Diamantes 10" (5000, 6666, 10000 and 20000 plants/ha) and two weed management strategies (chemical and physical) were compared. The parameters evaluated were plant height, stem diameter, shoot number per plant, palm heart units harvested, industrial yield and economic income. Besides, the effect on growth of weed competition was evaluated. Differences were observed among densities in regard to plant height and shoot number/plant at the age 9 month safter planting, being higher when planted at 6666 plants/ha). The higher planting densities gave the higher yields evaluated as number of stems harvested and number of exportable boxes during the first four months harvesting period.
\end{abstract}

Key words: peach palm, Bactris gasipaes, palm hearts, crop, densities.

\section{INTRODUCCIÓN}

Camacho y Soria (citados por Mora 1989) establecieron en el campo el primer estudio experimental de pejibaye para la producción de palmito a inicios de la década de los setentas, el cual consistió en una prueba de densidades de siembra, cuya recomendación inicial fue de 2.222 plantas por ha.
Otras evaluaciones de densidades de siembra para palmito con espinas se hicieron en los inicios del cultivo. Zamora (1984) evaluó entre 1980 y 1983, distancias de siembra con plantas a tallo simple "madre e hijo" y tallo doble "adulto y dos hijos" con bajas densidades, las cuales no superaban las 4.000 cepas/ha, en una época en que se comercializaba un palmito grueso. Vargas (1994) evaluó ocho densidades de siembra y obtuvo la

1 Recibido para publicación el 24 de junio del 2004.

2 Estación Experimental Los Diamantes, INTA-MAG. Costa Rica.

3 Facultad Agronomia, U.C.R.-San José, Costa Rica.

4 Facultad de Biología. U.C.R. San José, Costa Rica. 
mayor cantidad de palmitos en los primeros dos años de cosecha con la densidad más alta 6.666 plantas por ha $(1,5 \times 1,0 \mathrm{~m})$.

Bogantes et al. (1997) evaluaron 12 densidades de siembra en pejibaye con espinas y obtuvieron mayor rendimiento de palmito con las dos densidades más altas (13.333 y 10.000 plantas/ha). Las diferencias correspondientes fueron 76 y $42 \%$ más que la parcela testigo (5.000 plantas/ha). Estos autores sugieren que la primera distancia de $1,5 \times 0,5 \mathrm{~m}$ es manejable cuando se utiliza una variedad de palmito sin espinas porque, si posee espinas, éstas resultan molestas para el trabajador por la cercanía de las plantas y en ese caso es posible que sea necesario usar $2,0 \times 0,5 \mathrm{~m}$.

En Ecuador, Chalá (1991) evaluó ocho densidades de siembra en pejibaye para palmito y obtuvo los mejores rendimientos en producción de palmito durante el primer año con las densidades más altas (14.000 y 16.000 plantas/ha). Mientras que en Hawai, Clement et al. (1996) evaluaron las densidades de 3.333 (1,5 x $2 \mathrm{~m})$, $5.000(2 \times 1 \mathrm{~m})$ y 6.666 plantas por ha $(1,5 \times 1,0 \mathrm{~m})$ y no obtuvieron diferencias en peso del corazón de palmito, durante los primeros cuatro meses de cosecha.

Las recomendaciones sobre densidades de siembra han variado a través de los años. En un inicio se sembró a 1,5 m x 3,0 m lo que daba una densidad de 2.222 plantas por hectárea, también se recomendó producir palmito a distancias de $1,5 \times 1,5 \mathrm{~m} ; 2$ × $2 \mathrm{~m}$ y $2,5 \times 2,5$ $\mathrm{m}$, según fuera la topografía y la fertilidad del suelo (Fernández s. a.). Esas distancias se fueron modificando con el tiempo hasta llegar a 2 x $1 \mathrm{~m}$ para una densidad de 5.000 plantas, que se convirtió en la densidad estándar en el pejibaye con espinas hasta aproximadamente 1995.

Mora (1989) señala con respecto a las distancias de siembra que existen factores que obligan a variar las densidades, tales como: la fertilidad del suelo, el clima, las variedades, el uso de la maquinaria, el manejo agronómico o el mercado. Agrega que la introducción de nuevas variedades es un factor que empieza a jugar un papel importante en las densidades. Así, la presencia o ausencia de espinas influye en la selección de una u otra distancia.

Para Mora Urpí et al. (1997) el pejibaye para palmito se podría sembrar a alta densidad (20.000 plantas/ha), pero el espaciamiento, distribución geométrica y el número de tallos por planta son una importante consideración en la recomendación final. Además, Mora Urpí (1999) hace énfasis en que las innovaciones en pejibaye para palmito, con respecto a distancias de siembra, de- ben considerar la relación entre el número total de plantas (cepas) y el número de tallos por planta, de manera que se pueda manejar como criterio el total de tallos por hectárea en sustitución del número de cepas.

La expectativa de mayor producción con el uso de densidades de siembra más altas para producir palmito, su efecto en el control de las malezas y en el manejo, así como el potencial productivo del pejibaye sin espinas, el cual permite variar la distancia de siembra y brinda una nueva oportunidad de manejo (Mora Urpí et al. 1999), motivó la realización de este estudio cuyos objetivos fueron: la evaluación del efecto de cuatro densidades de siembra y dos estrategias de combate de malezas sobre el crecimiento y rendimiento inicial de plantas de pejibaye sin espinas para palmito.

\section{MATERIALES Y MÉTODOS}

\section{Área de estudio}

El estudio se realizó entre marzo del 2000 y mayo del 2001, en la Estación Experimental Los Diamantes del Ministerio de Agricultura y Ganadería, ubicada en Guápiles, cantón Pococí, provincia de Limón; a una altitud de $249 \mathrm{msnm}$, con una temperatura mínima promedio de $24{ }^{\circ} \mathrm{C}$ y una máxima promedio de $29{ }^{\circ} \mathrm{C}$. La precipitación promedio anual es de $4.500 \mathrm{~mm}$.

\section{Preparación del terreno y siembra}

Se realizó una pasada de arado, dos de rastra y se sembró en marzo del 2000. Se usaron plántulas con raíz desnuda, de pejibaye sin espinas de la variedad Diamantes 10 , con tres meses de germinadas y cuatro hojas juveniles. Los hoyos se hicieron con espeque y fue necesario efectuar resiembras una, cuatro y ocho semanas después de la siembra; la fertilización se hizo siguiendo las instrucciones hechas en el manual de recomendaciones del cultivo (Bogantes 1996).

\section{Tratamientos}

Los tratamientos evaluados fueron la combinación de cuatro distancias de siembra con dos estrategias de manejo de malezas para un total de ocho combinaciones factoriales (Cuadro 1).

La estrategia de deshierba física en las bandas consistió en eliminar las malezas con cuchillo, mientras que en el centro se hicieron dos chapeas con cuchillo y 
Cuadro 1. Tratamientos: distancias de siembra y estrategias de control de malezas. Guápiles, Costa Rica. 2000-2001.

\begin{tabular}{cccl}
\hline $\begin{array}{c}\text { Distancia/ } \\
\text { hilera } \\
(\mathbf{m})\end{array}$ & $\begin{array}{c}\text { Distancia/ } \\
\text { planta } \\
(\mathbf{m})\end{array}$ & $\begin{array}{c}\text { Densidad } \\
\text { (plantas/ha) }\end{array}$ & $\begin{array}{l}\text { Manejo } \\
\text { malezas }\end{array}$ \\
\hline 2,0 & 0,25 & 20.000 & Químico \\
2,0 & 0,50 & 10.000 & Químico \\
2,0 & 0,75 & 6.666 & Químico \\
2,0 & 1,00 & 5.000 & Químico \\
2,0 & 0,25 & 20.000 & Físico \\
2,0 & 0,50 & 10.000 & Físico \\
2,0 & 0,75 & 6.666 & Físico \\
2,0 & 1,0 & 5.000 & Físico \\
\hline
\end{tabular}

las siguientes con motoguadaña. El control químico se hizo con el herbicida glifosato (Round up 35,6 SL, 0,9$1,0 \mathrm{~kg} / \mathrm{ha} \mathrm{y} \mathrm{Ranger} \mathrm{Plus} 68 \%$ SG, 2,40 kg i.a./ha) según la cobertura y la altura de las malezas en el momento de la aplicación. Se usó una bomba de mochila marca Field King de 14 litros con una boquilla TJ 8002. El ancho de la banda a cada lado de la hilera fue de 0,25 metros y en el centro fue de 1,50 metros, lo que completa los $2 \mathrm{~m}$ entre hileras.

\section{Diseño experimental}

Se usó un diseño de parcelas divididas distribuidas en cinco bloques al azar. La estrategia de manejo de malezas se asignó a la parcela grande y las distancias de siembra entre plantas a la pequeña, para un total de 40 parcelas. Cada parcela pequeña midió $64 \mathrm{~m}^{2}$ y se usó una área útil de $48 \mathrm{~m}^{2}$; el área experimental total fue de $3.456 \mathrm{~m}^{2}$.

\section{Las variables evaluadas fueron:}

1) Altura y diámetro $(\mathrm{cm})$ de planta a los tres, seis y nueve meses después de la siembra (mds). mds.

2) Número de brotes (tallos) por planta a los nueve

3) Número de palmitos cosechados por parcela durante los primeros cuatro meses de cosecha. Se cortaron los tallos con un promedio de nueve $\mathrm{cm}$ de grosor, medidos en la base a $10 \mathrm{~cm}$ del suelo. El palmito se dejó con dos "cáscaras" (vainas) y con una longitud de 55 $\mathrm{cm}$ (incluye $9 \mathrm{~cm}$ de palmito caulinar y $46 \mathrm{~cm}$ de palmito foliar).
4) Número de trozos por palmito de nueve $\mathrm{cm}$ de longitud, peso fresco $(\mathrm{g})$ de palmito foliar y peso fresco (g) de palmito caulinar o "cabeza", de los primeros cuatro palmitos por parcela.

5) Peso en kilogramos de palmito foliar y el número de cajas estándar por parcela. El peso por parcela es el producto del número de palmitos por su peso fresco; mientras que el número de cajas estándar es ese cociente dividido entre $5,28 \mathrm{~kg}$ que es el peso del palmito drenado contenido en una caja estándar para exportación. Estas variables se estimaron en palmito fresco.

6) Ingresos por palmito fresco y por cajas producidas. Se hizo una estimación para comparar los ingresos totales en US \$, por producción de palmito y por cajas estándar producidas en los primeros cuatro meses de cosecha.

El análisis estadístico de las variables se hizo con el programa SAS. Para el ANDEVA de las variables altura y diámetro de planta, se analizaron los incrementos (crecimiento); para el palmito caulinar, los datos originales fueron transformados a raíz cuadrada de $\mathrm{x}$.

Para obtener información complementaria a partir de los 45 días después de la siembra (dds) y hasta los cuatro meses después de la siembra (mds), se evaluó el efecto de las malezas en el crecimiento de las plantas de pejibaye. Se manejó un bloque adicional con cuatro parcelas $\left(64 \mathrm{~m}^{2} \mathrm{c} / \mathrm{u}\right)$ por cada distancia.

De esas parcelas, dos se deshierbaron con cuchillo cada mes, y otras dos se dejaron a libre competencia. Se evaluaron tres plantas de las dos filas centrales por cada parcela para un total de 24 plantas en cada uno de los tratamientos.

Se evaluaron las siguientes variables: altura $(\mathrm{cm})$, diámetro $(\mathrm{cm})$, número de hojas y peso $(\mathrm{g})$ del follaje a los cuatro mds; y se compararon las medias de los tratamientos con y sin maleza para cada una de las variables con una prueba de t de Student.

\section{RESULTADOS}

\section{Incremento de altura, grosor y número de brotes por planta}

En los primeros seis meses de crecimiento (Cuadro 2) el incremento de altura en las plantas de pejibaye no fue diferente entre las distancias de siembra $(\mathrm{p} \leq 0,78)$, 
ni entre las estrategias de control de malezas $(\mathrm{p} \leq 0,19)$. El incremento de grosor del estípite, tampoco fue diferente entre las distancias $(\mathrm{p} \leq 0,89)$ ni entre las estrategias de control $(\mathrm{p} \leq 0,63)$.

A los nueve meses de la siembra (mds), se observaron diferencias en el incremento de altura de la planta entre las distancias de siembra $(\mathrm{p} \leq 0,02)$, el cual disminuyó conforme aumentó la distancia entre las plantas de palmito; en tanto que la estrategia de control de malezas, no afectó el incremento de la altura $(\mathrm{p} \leq 0,21)$. Hubo diferencias $(p \leq 0,04)$ en el incremento de grosor del tallo, entre las estrategias de control de malezas, y este fue mayor cuando se usó el combate químico, pero la distancia entre plantas no afectó esta variable $(\mathrm{p} \leq 0,31)$ (Cuadro 2).

Cuadro 2. Incrementos en altura y grosor del tallo en plantas de pejibaye a los seis y nueve mds (Medias de las distancias de siembra y estrategias de control de malezas). Guápiles, Costa Rica. 2000-2001.

\begin{tabular}{lcccc}
\hline Distancia (m) & \multicolumn{2}{c}{$\mathbf{6}$ mds $(\mathbf{c m})$} & \multicolumn{2}{c}{$\mathbf{9}$ mds $(\mathbf{c m})$} \\
& Altura & Grosor & Altura & Grosor \\
\hline \multirow{2}{*}{0,25} & 20,59 & 1,41 & 30,28 & 1,62 \\
0,50 & 20,12 & 1,43 & 29,64 & 1,78 \\
0,75 & 20,05 & 1,45 & 27,38 & 1,86 \\
1,00 & 19,16 & 1,36 & 25,02 & 1,71 \\
& $(\mathrm{p}=0,78)$ & $(\mathrm{p}=0,89)$ & $(\mathrm{p}=0,02)$ & $(\mathrm{p}=0,31)$ \\
Estrategia & & & & \\
Física & 19,44 & 1,40 & 26,92 & 1,60 \\
Química & 20,52 & 1,44 & 29,23 & 1,89 \\
& $(\mathrm{p}=0,19)$ & $(\mathrm{p}=0,63)$ & $\mathrm{p}=(0,21)$ & $(\mathrm{p}=0,04)$ \\
\hline
\end{tabular}

El número de brotes por planta, nueve mds, aumentó con la distancia entre plantas hasta $0,75 \mathrm{~m}$, luego disminuyó $(\mathrm{p} \leq 0,01)$.

No hubo diferencias en el número de brotes entre estrategias de control de malezas $(\mathrm{p} \leq 0,47)$ (Cuadro 3 ).

\section{Producción de palmito}

A los 14 meses de edad, y con solo cuatro meses de cosecha, se había cosechado en promedio un $81 \%$ de las parcelas sembradas a $0,25 \mathrm{~m}$ entre plantas $(16277$ palmitos/ha); el $94 \%$ en las parcelas a $0,5 \mathrm{~m}(9.407$ palmitos/ha); un $105 \%$ (un 5\% del siguiente ciclo) en las parcelas a $0,75 \mathrm{~m}$ (7.009 palmitos/ha) y el $99 \%$ de las plantas sembradas a $1 \mathrm{~m}$ entre plantas (4.963 palmito/ha). Durante ese período se notó un marcado efecto
Cuadro 3. Brotes por cepa \pm error estándar, según la distancia de siembra y la estrategia de control de malezas, nueve mds. Guápiles, Costa Rica. 2000-2001.

\begin{tabular}{cccc}
\hline $\begin{array}{c}\text { Distancia entre } \\
\text { planta (m) }\end{array}$ & \multicolumn{2}{c}{ Control de malezas } & Medias \\
Físico & Químico & $(\mathbf{p = 0 , 0 1 )}$ \\
\hline 0,25 & $1,15 \pm 0,35$ & $0,80 \pm 0,27$ & 0,98 \\
0,50 & $1,20 \pm 0,34$ & $1,40 \pm 0,41$ & 1,30 \\
0,75 & $2,20 \pm 0,48$ & $1,95 \pm 0,51$ & 2,08 \\
1,00 & $1,30 \pm 0,36$ & $0,95 \pm 0,38$ & 1,12 \\
Medias $(\mathrm{p}=0,47)$ & 1,46 & 1,27 & \\
\hline
\end{tabular}

de la distancia entre plantas sobre el número de palmitos cosechados por parcela $(\mathrm{p} \leq 0,0001)$. A mayor distancia entre plantas (menor densidad) disminuyó la producción de palmitos con una tendencia curvilínea (Figura 1) $(\mathrm{p} \leq 0,0001)$.

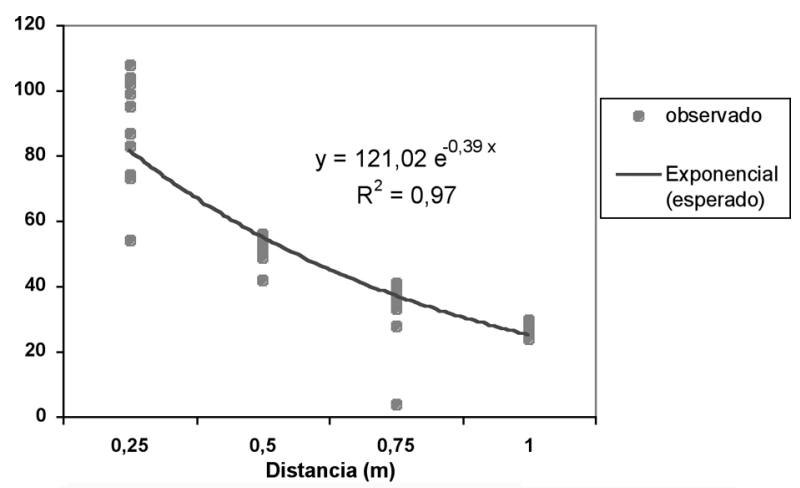

Figura 1. Producción de palmito en cuatro densidades de siembra (primeros cuatro meses de cosecha). Guápiles, Costa Rica. 2000-2001.

La relación entre distancias de siembra y la producción de palmitos e ingresos por hectárea es consecuente con lo anterior, ambos disminuyeron conforme aumentó la distancia entre plantas. Los ingresos más altos se obtienen con la distancia $0,25 \mathrm{~m}$ entre plantas, mientras que los más bajos con $1 \mathrm{~m}$ entre plantas (Cuadro 4). No hubo efecto de las estrategias de combate de malezas $(\mathrm{p} \leq 0,95)$ sobre la producción de palmitos.

\section{Rendimiento industrial}

La distancia de siembra entre plantas afectó el número de trozos por palmito $(\mathrm{p} \leq 0,04)$, a menor distancia entre plantas fue mayor el número de trozos de palmito foliar (Cuadro 5); pero incidió negativamente 
Cuadro 4. Rendimiento de palmito e ingresos brutos por ha, según distancia en cuatro meses de cosecha . Guápiles, Costa Rica. 2000-2001.

\begin{tabular}{cccccc}
\hline $\begin{array}{c}\text { Distancia } \\
(\mathbf{m})\end{array}$ & $\begin{array}{c}\text { \% cosecha } \\
\text { por } \\
\text { parcela }\end{array}$ & parcela & ha & \multicolumn{3}{c}{$\begin{array}{c}\text { Unidades/ Ingreso/ Diferencia } \\
\text { ha }\end{array}$} & $(\%)$ \\
& & & & $($ US \$) & \\
\hline & & & & & \\
0,25 & 81 & 87,9 & 16.277 & 2.604 & 0 \\
0,50 & 94 & 50,8 & 9.407 & 1.505 & -42 \\
0,75 & $105^{*}$ & 34,7 & 7.009 & 1.122 & -57 \\
1,00 & 99 & 26,8 & 4.963 & 794 & -70 \\
\hline
\end{tabular}

Precio por unidad de palmito US \$ 0,16 * 5\% del segundo ciclo de cosecha

Cuadro 5. Número de trozos ( \pm error estándar) de palmito foliar por unidad, según distancia de siembra y estrategia de control de malezas. Guápiles, Costa Rica. 2000-2001.

\begin{tabular}{cccc}
\hline $\begin{array}{c}\text { Distancia entre } \\
\text { plantas }(\mathbf{m})\end{array}$ & \multicolumn{2}{c}{ Control de malezas } & Medias \\
Químico & $(\mathbf{p = 0 , 0 4 )}$ \\
\hline \multirow{2}{*}{0,25} & $3,05 \pm 0,22$ & $3,15 \pm 0,17$ & 3,10 \\
0,50 & $3,30 \pm 0,18$ & $3,35 \pm 0,15$ & 3,32 \\
0,75 & $2,60 \pm 0,22$ & $3,15 \pm 0,15$ & 2,88 \\
1,00 & $3,05 \pm 0,20$ & $2,80 \pm 0,16$ & 2,92 \\
\multirow{2}{*}{ Medias } & 3,11 & 3,00 & \\
\hline
\end{tabular}

sobre el peso de cada trozo, el cual disminuyó en forma lineal con la reducción de la distancia entre plantas $\left(\mathrm{R}^{2}=0,99\right)$ (Figura 2).

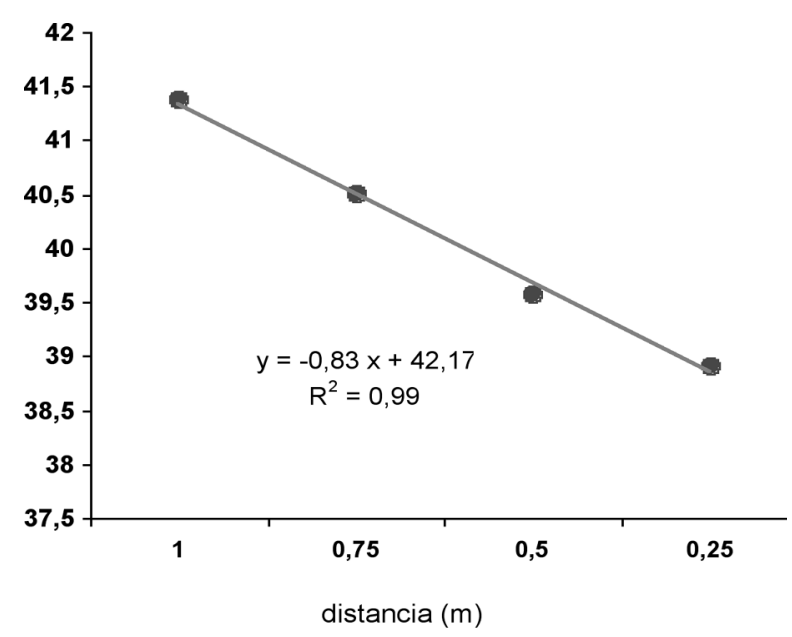

Figura 2. Peso promedio del trozo de palmito foliar $(9 \mathrm{~cm}) \mathrm{se}-$ gún distancia de siembra. Guápiles, Costa Rica.2001.
Con respecto al peso fresco de palmito foliar por unidad (Cuadro 6) no hubo diferencias entre distancias de siembra, la mayor longitud del palmito compensó la disminución de peso señalado anteriormente. No hubo diferencias entre estrategias de control sobre el peso de palmito foliar ( $\mathrm{p} \leq 0,47$ y 0,59$)$ ni sobre el palmito caulinar o "cabeza" (p $\leq 0,42$ y p $\leq 0,31)$ (Cuadro 7 ).

Cuadro 6. Peso fresco (g) de palmito foliar ( \pm error estándar), según distancia de siembra y estrategia de control de malezas. Guápiles, Costa Rica. 2000-2001.

\begin{tabular}{cccc}
\hline $\begin{array}{c}\text { Distancia entre } \\
\text { plantas (m) }\end{array}$ & \multicolumn{2}{c}{ Control de malezas } & Medias \\
& Químico & $(\mathbf{p = 0 , 4 7 )}$ \\
\hline 0,25 & $119,24 \pm 12,39$ & $122,08 \pm 7,95$ & 120,66 \\
0,50 & $134,72 \pm 8,88$ & $128,11 \pm 7,61$ & 131,42 \\
0,75 & $103,47 \pm 11,18$ & $129,89 \pm 9,73$ & 116,68 \\
1,00 & $117,66 \pm 12,48$ & $124,02 \pm 10,32$ & 120,84 \\
Medias $(0,59)$ & 118,77 & 126,03 & \\
\hline
\end{tabular}

Cuadro 7. Peso fresco ( \pm error estándar) de palmito caulinar (g), según distancia de siembra y estrategia de control de malezas. Guápiles, Costa Rica. 2000-2001.

\begin{tabular}{cccc}
\hline $\begin{array}{c}\text { Distancia entre } \\
\text { plantas (m) }\end{array}$ & \multicolumn{2}{c}{ Control de malezas } & Medias \\
Químico & $(\mathbf{p = 0 , 4 2 )}$ \\
\hline 0,25 & $63,04 \pm 5,38$ & $63,96 \pm 7,59$ & 63,50 \\
0,50 & $54,24 \pm 5,08$ & $59,21 \pm 7,29$ & 56,72 \\
0,75 & $55,58 \pm 6,83$ & $58,33 \pm 7,20$ & 56,96 \\
1,00 & $60,48 \pm 6,18$ & $82,97 \pm 19,81$ & 71,73 \\
Medias (p=0,31) & 58,34 & 66,12 & \\
\hline
\end{tabular}

Es natural que si el rendimiento en kg por hectárea de palmito foliar aumentó con la mayor densidad, el número de cajas de estándar de palmito y los ingresos por ha de palmito exportable, aumentara proporcionalmente (Cuadro 8).

\section{Crecimiento de plantas a libre competencia con las malezas}

Como era de esperar, en el bloque adicional, la altura y el grosor del tallo, fueron muy superiores en las plantas con deshierba física ( $\mathrm{p} \leq 0,0001$ ) con respecto 
Cuadro 8. Rendimiento industrial de palmito foliar (peso fresco) e ingresos brutos por ha, según distancia. Primeros cuatro meses de cosecha. Guápiles, Costa Rica. 2000-2001.

\begin{tabular}{ccccccc}
\hline $\begin{array}{c}\text { Distancia } \\
(\mathbf{m})\end{array}$ & $\begin{array}{c}\text { Peso (g) } \\
\text { Unidad }\end{array}$ & kg/parcela & kg/ha & *Cajas/ha & $\begin{array}{c}\text { Ingreso/ha } \\
* * \text { US }(\$)\end{array}$ & $\begin{array}{c}\text { Diferencia } \\
(\%)\end{array}$ \\
\hline 0,25 & 120,66 & 10,61 & 1.964 & 372 & 6.696 & 0 \\
0,50 & 131,42 & 6,68 & 1.236 & 234 & 4.215 & -37 \\
0,75 & 116,68 & 4,05 & 818 & 155 & 2.788 & -58 \\
1,00 & 120,84 & 3,24 & 600 & 114 & 2.045 & -69 \\
\hline
\end{tabular}

* Peso de caja equivalente o estándar 5,28 kg ** caja estándar = US \$ 18.

a las plantas a libre competencia (testigo). También el número de hojas y peso fresco del follaje fue considerablemente mayor en las plantas libres de malezas con respecto al testigo enmalezado ( $\mathrm{p} \leq 0,0001)$ (Cuadro 9).

\section{DISCUSIÓN}

\section{Incremento de altura, grosor y número de estípites por planta}

Como es natural, la velocidad en el crecimiento de las plantas durante los primeros seis meses del cultivo fue menor a la que se dio entre los seis y nueve meses. Antes de los seis meses no hubo competencia entre plantas por su poco desarrollo. Mora et al. (1999) señalan que las plantas de pejibaye para palmito durante los primeros meses de desarrollo, no ocupan todo el terreno con sus raíces ni toda el área con su follaje, lo cual permite un crecimiento normal, sin competencia. Esto explica que no se dieran diferencias entre las distancias de siembra en las variables de crecimiento, grosor y número de estípites en los primeros seis meses. A los nueve meses de edad del cultivo, se hizo aparente el macollamiento, las plantas alcanzaban mayor altura, el número de hojas aumentó y con ello el autosombreo que pudo incidir en el incremento de la altura. En una evaluación de la palma
Phoenix roebelenii, Jiménez (1998) observó a los 21 meses mayor altura en las plantas sembradas a más densidad (12.500, 15.625, 25.000 y 31.250 planta/ha).

Con respecto al macollamiento, en general la cantidad de brotes producida hasta los 9 mds fue baja, situación normal en los estípites "madres", cuya producción se estimula con la poda ocasionada por la cosecha. Adicionalmente, la producción de brotes por los materiales sin espinas parecen ser más susceptibles a luminosidad baja que las plantas con espinas pero esto no limita la producción, sobre todo a altas densidades por crecer con más rapidez (Mora et al. 1999).

\section{Producción de palmito}

La cosecha se realizó a los 10 meses después de la siembra, lo cual era una meta de precocidad propuesta para nuevas variedades (Mora 1999). En general esos altos porcentajes de cosecha en los primeros cuatro meses, indican un crecimiento rápido y homogéneo del cultivo. Esto probablemente se logró al sembrar las plantas en el campo a muy temprana edad, situación que difiere notoriamente de lo encontrado por Clement et al. (1996) en Hawaii, quienes durante los primeros cuatro meses, cosecharon solo el $28 \%$ de cada parcela.

Cuadro 9. Promedios ( \pm error estándar) de altura de planta, grosor del tallo, número de hojas y peso fresco de follaje en plantas con y sin malezas 4,5 mds. Guápiles, Costa Rica. 2000-2001.

\begin{tabular}{lcccc}
\hline Tratamiento & Altura $(\mathbf{c m})$ & Grosor $(\mathbf{c m})$ & \# hojas & Follaje $(\mathbf{g})$ \\
\hline Con malezas & $5,92 \mathrm{a} \pm 0,70$ & $0,45 \mathrm{a} \pm 0,03$ & $2,46 \mathrm{a} \pm 0,15$ & $6,25 \mathrm{a} \pm 0,76$ \\
Sin malezas & $22,65 \mathrm{~b} \pm 1,28$ & $1,73 \mathrm{~b} \pm 0,09$ & $6,54 \mathrm{~b} \pm 0,15$ & $87 \mathrm{~b} \pm 9,92$ \\
T de Student & $(\mathrm{p}<0,0001)$ & $(\mathrm{p}<0,0001)$ & $(\mathrm{p}<0,0001)$ & $(\mathrm{p}<0,0001)$ \\
\hline
\end{tabular}


Respecto al efecto de la densidad de siembra en la producción de palmitos, se obtiene una regresión curvilínea que indica que el aumento en la distancia entre plantas reduce la producción del número de palmitos. Esa tendencia coincide con los resultados obtenidos por Zamora (1984), Vargas (1994), Bogantes et al. (1997), en estudios realizados en palmito con espinas de la variedad Utilis-Tucurrique.

Una de las principales ventajas de la alta densidad según Mora et al. (1999) es obtener altos rendimientos en los primeros meses de producción, lo cual justificaría la mayor inversión inicial. En este caso si observamos los rendimientos por hectárea es claro que con altas densidades, la producción de palmitos en los primeros cuatro meses, fue superior en las parcelas sembradas con la menor distancia $(0,25 \mathrm{~m}$ entre planta) y por lo tanto los ingresos en US \$ por hectárea también aumentaron proporcionalmente. Se obtuvo un $70 \%$ de aumento en producción e ingresos a favor de las parcelas con menor distancia entre plantas. En ese sentido Quevedo y Reyes (2000) consideran que en palmito de pejibaye la producción de tallos a bajas densidades es subóptima durante los primeros años.

\section{Rendimiento industrial}

La mayor altura de las plantas sembradas a menor distancia, tuvo un efecto directo en el número de trozos de palmito foliar, las cuales produjeron mayor número de trozos de palmito foliar, aunque estos fueron más delgados y de menor peso. Lo anterior tiene importancia si se piensa en la tendencia industrial de preferir un palmito más delgado para un mercado en el cual el palmito compite con el espárrago (Mora 1989).

El peso total de palmito foliar producido por hectárea, depende principalmente del número de palmitos producidos. En ese sentido, si observamos el Cuadro 8, se nota que la producción de cajas de palmito así como los ingresos totales por su venta, obtenidos con la distancia de $0,25 \mathrm{~m}$ superan en $69 \%$ los rendimientos e ingresos obtenidos que cuando se siembra a $1 \mathrm{~m}$. Lo indicado no considera el palmito caulinar, el cual aún no es bien aprovechado por la industria, a pesar de ser un componente importante del peso del palmito total.

\section{Crecimiento de plantas a libre competencia con las malezas}

La libre competencia entre las plantas de pejibaye y las malezas incidió directamente en el crecimiento del cultivo. Bogantes y Aguero (1998) indican que en cultivos para palmito no existen estudios sobre períodos críticos de competencia con malezas. Hinestroza (1996), indica que el control de malezas en el primer año de establecimiento es especialmente importante.

Pérez et al. (1991) encontraron que plantas jóvenes de pejibaye, para producción de fruta, fueron sensibles a la competencia con leguminosas y crecieron mejor cuando estuvieron sin la cobertura. La competencia con leguminosas, afectó la altura, el grosor del tallo y la producción de hojas. En la evaluación que se realizó en la presente investigación, paralela a la prueba principal, fue evidente que en un período de 2,5 meses de crecimiento del cultivo a libre competencia con las malezas, los valores de crecimiento obtenidos fueron muy inferiores a aquellos sin malezas. Comparativamente, durante ese período la competencia causó disminuciones de aproximadamente $74 \%$ en la altura, $75 \%$ en el grosor del tallo, $63 \%$ en el número de hojas y $72 \%$ en el peso total del follaje.

En conclusión, a la edad de nueve meses después de la siembra la densidad afectó variables como la altura de la planta mientras que la estrategia de control de malezas incidió en el grosor del tallo. El número de brotes por cepa nueve mds fue mayor en las parcelas con plantas sembradas a $0,75 \mathrm{~m}$, lo que refleja el efecto positivo de la luz sobre el macollamiento de la cepa.

Además, la altura de las plantas, el grosor del tallo, el número de hojas y el peso fresco del follaje de las plantas fue afectado negativamente por la libre competencia con las malezas.

El rendimiento en $\mathrm{kg}$ de palmito foliar por hectárea, aumentó considerablemente con la disminución en las distancias de siembra, y así los ingresos totales más altos por producción de palmitos y cajas exportables se obtuvieron con la mayor densidad de siembra, mientras que los más bajos se dieron con la menor densidad.

\section{LITERATURA CITADA}

BOGANTES, A. 1996. Recomendaciones para la siembra y manejo de palmito de pejibaye (Bactris gasipaes B. K.). Estación Experimental Los Diamantes. Guápiles, Costa Rica. 12 p.

BOGANTES, A; AGÜERO, R. 1998. Manejo de malezas en pejibaye. Agronomía Mesoamericana 9 (2):139-146.

BOGANTES, A; MORA, J. ; ARROYO, C. 1997. Densidades de siembra. In: J. Mora, J. Gainza (eds.). Memoria Curso Nacional sobre palmito de pejibaye (Bactris gasipaes). San José, Costa Rica. Universidad de Costa Rica. 5 p. 
CLEMENT, C; MANSHARDT, J; DEFRANK, J; CATHERINE, G; NAGAI, N. 1996. Introduction of pejibaye for heart-of-palm in Hawaii. Hort Science 31(5): 765-768.

CHALA, V. 1991. Evaluación de ocho densidades de siembra de Bactris gasipaes H.B.K.para producción de palmito en la región amazónica ecuatoriana. In: J. Mora, L. T. Szott, M. Murillo, V. Patiño (eds.). IV Congreso internacional sobre Biología, Agronomía e Industrialización del Pijuayo. Editorial U.C.R. San José, Costa Rica. p 255-265.

FERNANDEZ, B. s.a. El pejibaye. Serie de boletines. Boletín Técnico para el agricultor. Costa Rica. Proyecto ANAI. No. 3. 29 p.

HINESTROZA, A. 1996. Manejo de malezas en el establecimiento de una plantación de pejibaye (Bactris gasipaes) para palmito. Tesis Ing. Agr., EARTH. Costa Rica. p. 18-38.

JIMENEZ, K. 1998. Efecto de la densidad de siembra sobre el crecimiento de Phoenix roebelleni (Dwarf date palm) para follaje. Boletín Técnico Fabio Baudrit 31(1): 31-36.

MORA, URPÍ, J. 1989. Densidades de siembra para producción de palmito. Universidad de Costa Rica. Serie técnica Pejibaye 1 (1): 10-12 .

MORA, URPÍ, J.; WEBER, J.; CLEMENT, CH. 1997. Peach and palm. Bactris gasipaes Kunth. Promoting the conservation and use of underutilized and neglected crops. 20. Institute of plants genetics and Crop Plant
Research, Gaterslebem/International Plant Genetic Resources Institute, Rome, Italy. 44 p.

MORA, URPÍ, J. 1999. Bases para evaluar comercialmente futuras Innovaciones en el cultivo. In: J. Mora; J. Gainza (eds.). Palmito de pejibaye (Bactris gasipaes K.) su cultivo e industrialización. San José, Costa Rica. Editorial U.C.R. p. 48-50.

MORA, URPÍ, J.; BOGANTES, A.; ARROYO, C. 1999. Cultivares de pejibaye para palmito. In: J. Mora y J. Gainza (eds.). Palmito de pejibaye (Bactris gasipaes K.). su cultivo e industrialización. Editorial U.C.R. San José, Costa Rica. pp. 41-47.

PEREZ, J. M; SZOTT, L.T; AREVALO, L.A. 1991. Pijuayo con cobertura de legumnosas. In: J. Mora, L. T. Szott, M. Murillo y V. Patiño (eds.). IV Congreso internacional sobre Biología, Agronomía e Industrialización del Pijuayo. Editorial U.C.R. San José, Costa Rica. p. 309-316.

QUEVEDO, E.; REYES, R. 2000. Densidad de siembra. In: Reyes, R.; Peña, E.; Gómez, J. (eds.). El cultivo de chontaduro (Bactris gasipaes K.) para palmito. CORPOICA. San Andrés de Tumaco, Colombia. p. 44-47.

VARGAS. A. 1994. Evaluación de ocho densidades de siembra en pejibaye para palmito (Bactris gasipaes H.B.k.) en el Atlántico de Costa Rica. CORBANA 19 (42): 11-16.

ZAMORA, C. 1984. Densidades de siembra de pejibaye para palmito con tallo simple y tallo doble. Sexto Informe de labores 1983-1984. ASBANA. pp. 75-80. 